\title{
23. Immunoreactive Atrial Natriuretic Peptide in the Heart and Plasma of the Toad, Bufo japonicus formosus, during the Breeding Season
}

\author{
By Haruko Uemura, *) Mitsuhide Naruse,**) Tohru Hirohama,*) \\ Sumio NAKamURa,*) and Tomoji AoTo*)
}

(Communicated by Yoshimasa NishikAwA, M. J. A., April 12, 1989)

Introduction. Mammalian atrial cardiocytes have recently been found to produce a family of peptides, termed atrial natriuretic peptides (ANP), which perform biological functions such as vasodilation, natriuresis and diuresis. In submammalian vertebrates, immunoreactive ANP (ir-ANP) has been shown present in the cardiocytes of several species of each class of vertebrates (Chapeau et al., 1985; Reinecke et al., 1985, 1987; Netchitailo et al., 1986; Hirohama et al., 1988). In the case of amphibians, the presence or ir-ANP in frog atrial and ventricular cardiocytes has been reported (Chapeau et al., 1985; Reinecke et al., 1985; Netchitailo et al., 1986), and the primary structure of frog ANP has been demonstrated (Sakata et al., 1988). However, research on the function of these peptides has been conducted only to a limited extent; frog atrial and ventricular extracts have been found to lead to the expression of natriuretic and diuretic activities in rats (De Bold and Salerno, 1983). In the present study, the plasma level of ir-ANP and secretory activity of cardiocytes were examined for possible differences between aquatic and terrestrial life in breeding and non-breeding seasons, respectively, in toads.

Materials and methods. Animals. Adult specimens of Bufo japonicus formosus were collected from a pond in April (spring toads) and from a field in August (summer toads). The animals were kept for one or two days in a sink and occasionally wetted.

Karyometric study and immunohistochemistry. Three spring and summer toads each were used. The hearts, following pithing of the animals, were dissected out, fixed in Bouin's solution and made into $6 \mu \mathrm{m}$ thick sagittal paraffin sections. The sections were stained with hematoxylin and eosin and subsequently used for karyometric study. Some sections were immunostained. Cardiocyte activity in the right atrium and ventricle was expressed in terms of the size of the nucleus. The nucleus of a cardiocyte is very elongated, measuring as much as $55 \mu \mathrm{m}$, so that its whole length cannot be measured on a $6 \mu \mathrm{m}$-thick section. Thus, in this study, activity was expressed in terms of the width of a cardiocyte nucleus in longitudinally-sectioned trabeculae. For this purpose, one hundred nuclei from the right atrium and ventricle each were measured for width, and the mean value and standard error were calculated from individually obtained mean values of three toads.

Immunohistochemistry was performed according to the peroxidase-antiperoxidase (PAP) method (Sternberger et al., 1970), using anti- $\alpha$-hANP antiserum

*) Biological Laboratory, Kanagawa Dental College, Yokosuka 238, Japan.

**) Institute of Clinical Endocrinology, Department of Medicine, Tokyo Women's Medical College, Tokyo 162, Japan. 
(1:660; Peptide Inst., Osaka), normal goat serum (1:20; Polysciences, Inc., Warrington), goat anti-rabbit IgG serum (1:100 ; Polysciences Inc.) and peroxidaseanti-peroxidase complex (1:100; Dakopatts, Denmark). The specificity of the immunohistochemical staining was confirmed by replacing the antiserum with that preabsorbed by excess hANP $(10 \mu \mathrm{g} / \mathrm{ml}$ of hANP antiserum diluted $1: 660)$ at $4{ }^{\circ} \mathrm{C}$ for $48 \mathrm{hr}$. This procedure resulted in the complete loss of immunoreactivity in cardiocytes in all cases.

Blood collection and radioimmunoassay (RIA). Four spring and summer toads each were used. After pithing the animals, blood samples were taken from the conus arteriosus with a heparinized syringe and each sample was transferred to a chilled tube. The plasma was separated by centrifugation at $4^{\circ} \mathrm{C}$. ANP was extracted and its amount determined by RIA as described previously (Naruse et al., 1986), except that anti- $\alpha$-hANP antiserum was used (Yoshinaga et al. 1986). The dilution curve obtained with the plasma extracts essentially paralleled the standard curve.

Statistical analysis. Data were analysed by the Student's $t$-test.

Results. Histological and immunohistochemical observations. The mean values and standard errors for nuclear size in cardiocytes of the right atrium (AtN) and ventricle $(\mathrm{VenN})$ were $4.4 \pm 0.2 \mu \mathrm{m}$ and $3.8 \pm 0.1 \mu \mathrm{m}$, respectively, in the srping toads $(\mathrm{n}=3)$ and $2.7 \pm 0.1 \mu \mathrm{m}$ and $3.0 \pm 0 \mu \mathrm{m}$, respectively, in the summer toads $(n=3)$. The difference in nuclear size between the spring and summer toads is highly significant $(p<0.01)$ statistically in both the atrium and ventricle. The atrial cardiocyte nucleus was larger than that of the ventricular cardiocyte in the spring toads, but smaller in the summer toads. Thus, the ratio of AtN to

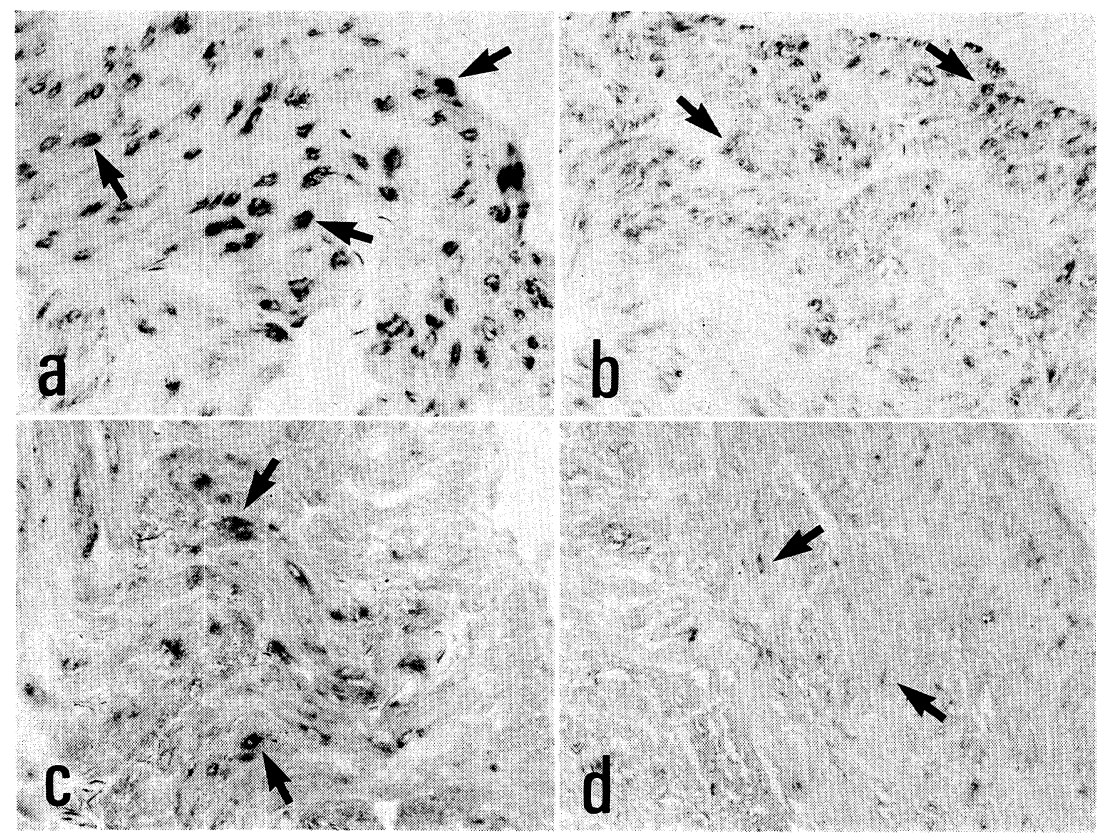

Fig. 1. Atria (a, b) and ventricles (c, d) of spring (a, c) and summer (b, d) toads. At all times, ANP-immunoreactivity (arrows) was stronger in the atrium than in the ventricle, and the tissues of spring toads were more immunoreactive than those of summer toads. $\times 150$. 
VenN changed remarkably to 1.15 in the spring toads from 0.91 in the summer toads.

Immunohistochemically, atrial cardiocytes in the spring toads showed stronger immunoreactivity than did those in the summer toads (Fig. 1, a, b). In the ventricles, some cardiocytes showed positive reaction in the spring toads, while only a few cells indicated trace reaction in the summer toads (Fig. 1, c, d).

$A N P$ in plasma. The plasma ir-ANP levels were $20.5 \pm 5.9 \mathrm{pg} / \mathrm{ml}$ (Mean $\pm \mathrm{SE}$, $\mathrm{n}=4)$ in the spring toads and $5.4 \pm 1.7 \mathrm{pg} / \mathrm{ml}(\mathrm{n}=4)$ in the summer toads, respectively. The difference between these two values is statistically significant $(p<0.05)$.

Discussion. The cardiocytes in both the atrium and ventricle of the spring toads had larger nuclei and showed stronger immunoreactivity than did those of the summer toads. Plasma ir-ANP levels were also higher in the spring than summer toads. It thus appears that both atrial and ventricular cardiocytes are activated and contribute to high levels of circulating ir-ANP. Nuclear enlargement was more prominent in the atrial cardiocytes $(163 \%)$ than ventricular cardiocytes $(127 \%)$ in the spring toads, thus indicating that the atrial cardiocytes are primarily responsible for maintaining the high levels of circulating ir-ANP.

The biological significance of higher plasma ir-ANP in the spring toads is not clear at present, but it may possibly serve some physiological roles such as regulation of body fluid and sodium balance in temporary aquatic life during the breeding season. In this context, the administration of frog atrial and ventricular extracts to rats led to the expression of diuretic and natriuretic activities (De Bold and Salerno, 1983). Further study should be carried out to elucidate the physiological functions of ANP in relation to breeding of toads.

Acknowledgments. The present study was supported in part by Grants-inAid for Scientific Research from the Japanese Ministry of Education, Science and Culture.

\section{References}

Chapeau, C. et al.: Localization of immunoreactive synthetic atrial natriuretic factor (ANF) in the heart of various animal species. J. Histochem. Cytochem., 33, 541-550 (1985).

De Bold, A. J., and Salerno, T. A.: Natriuretic activity of extracts obtained from hearts of different species and from various rat tissues. Can. J. Physiol. Parmacol., 61, 127$130(1983)$.

Hirohama, T. et al.: Atrial natriuretic peptide (ANP)-immunoreactivity and ultrastructures of cardiocytes in fish. Zool. Sci., 5, 831-843 (1988).

Naruse, M. et al.: Immunoreactive $\alpha$-human atrial natriuretic polypeptide in human plasma. Peptides, 7, 141-145 (1986).

Netchitailo, P. et al.: Localization and characterization of atrial natriuretic factor (ANF)-like peptide in the frog atrium. ibid., 7, 573-579 (1986).

Reinecke, M., Nehls, M., and Forssmann, W. G.: Phylogenetic aspects of cardiac hormones as revealed by immunocytochemistry, electronmicroscopy, and bioassay. ibid., 6 (suppl. 3), 321-331 (1985).

Reinecke, M., Betzler, D., and Forssmann, W. G.: Immunocytochemistry of cardiac polypeptide hormones (cardiodilatin/atrial natriuretic polypeptide) in brain and hearts of Myxine glutinosa (Cyclostomata). Histochemistry, 86, 233-239 (1987).

Sakata, J., Kangawa, K., and Matsuo, H.: Identification of new atrial natriuretic peptides in frog heart. Biochem. Biophys. Res. Comm., 155, 1338-1345 (1988).

Sternberger, L. A. et al.: The unlabeled antibody enzyme method of immunohistochemistry. J. Histochem. Cytochem., 18, 315-333 (1970).

Yoshinaga, K. et al.: Determination of atrial natriuretic polypeptide (ANP) in human plasma. Biomed. Res., 7, 173-179 (1986). 